\title{
Micro-computed tomography assessment of dentinal microcracks after the preparation of curved root canals with rotary and reciprocal systems
}

\author{
Yazdan Shantiaee ${ }^{A, E, F}$, Babak Zandi ${ }^{A}$, Shiva Shojaeian ${ }^{B}$, Niloofar Mortezapour ${ }^{B}$, Fatemeh Soltaninejad ${ }^{A-D}$ \\ Department of Endodontics, School of Dentistry, Shahid Beheshti University of Medical Sciences, Tehran, Iran \\ A - research concept and design; $\mathrm{B}$ - collection and/or assembly of data; $\mathrm{C}$ - data analysis and interpretation; \\ $D$ - writing the article; $E$ - critical revision of the article; $F$ - final approval of the article
}

Address for correspondence

Fatemeh Soltaninejad

E-mail: Fsoltani69@gmail.com

Funding sources

None declared

Conflict of interest

None declared

Acknowledgements

The authors would like to thank Dr. N. Zargar for her support in implementing this project at the beginning of the COVID-19 pandemic, the Department of Oral and Maxillofacial Radiology of Shahid Beheshti University of Medical Sciences, Tehran, Iran, for CBCT scans, and the Tehran University of Medical Sciences Preclinical Core Facility (TPCF) for micro-CT scans.

Received on December 4, 2020

Reviewed on February 28, 2021

Accepted on March 9, 2021

Published online on December 31, 2021

Cite as

Shantiaee Y, Zandi B, Shojaeian S, Mortezapour N, Soltaninejad F. Micro-computed tomography assessment of dentinal microcracks after the preparation of curved root canals with rotary and reciprocal systems. Dent Med Probl. 2021;58(4):515-523. doi:10.17219/dmp/134149

DOI

$10.17219 / \mathrm{dmp} / 134149$

Copyright

○) 2021 by Wroclaw Medical University

This is an article distributed under the terms of the

Creative Commons Attribution 3.0 Unported License (CC BY 3.0)

(https://creativecommons.org/licenses/by/3.0/).

\begin{abstract}
Background. Root canal preparation with nickel-titanium (NiTi) instruments may lead to the formation of microcracks in the root canal wall. Vertical root fractures may initiate from dentinal cracks, and eventually necessitate tooth extraction.

Objectives. This study aimed to assess the effect of the instrumentation of curved root canals of mandibular molars with the 2Shape (2S) sequential rotary, EdgeFile ${ }^{\circledR}$ X1 (EFX1) reciprocating and NeoNiTi (NN) rotational single-file systems on the formation of dentinal microcracks with the use of micro-computed tomography (micro-CT).
\end{abstract}

Material and methods. Thirty curved mandibular molar root canals were instrumented with the 25 , EFX1 and NN systems (10 in each group). The teeth underwent micro-CT before and after instrumentation. Next, the pre-instrumentation and post-instrumentation cross-sectional images were evaluated and compared for the detection of dentinal microcracks. The number of microcracks in each group was calculated and reported as percentage. The data was analyzed using the McNemar's test with the IBM SPSS Statistics for Windows software, v. $25.0(a=0.05)$.

Results. Out of the 29,280 cross-sectional images evaluated in this study, $11.5 \%$ showed dentinal microcracks $(n=3,362)$. On the post-instrumentation images, the frequency percentage of microcracks was $12.0 \%(n=585)$ in the 25 group, $8.8 \%(n=402)$ in the EFX1 group and $13.3 \%(n=694)$ in the NN group. All of the microcracks detected on the post-instrumentation images were also present on the preinstrumentation images and no new microcracks were formed after root canal instrumentation with the aforementioned systems.

Conclusions. Root canal instrumentation with the 2S, EFX1 and NN systems did not result in the formation of new dentinal microcracks.

Keywords: root canal preparation, microcracks, dentinal defect, micro-computed tomography, curved root canal 


\section{Introduction}

The main goal of the chemomechanical preparation of the root canal system is to eliminate microorganisms, pulpal tissue and debris, and to shape the root canal in order to create adequate space for the root filling material. ${ }^{1}$ Nickel-titanium (NiTi) rotary systems with improved properties are produced by several manufacturers to achieve these goals. $\mathrm{NiTi}$ systems have numerous advantages over stainless-steel hand files, such as a higher flexibility and a shorter working time. However, root canal preparation with these instruments may lead to the formation of microcracks in the root canal wall. ${ }^{2,3}$ Microcracks may occur as a result of the distribution of lateral forces in root canal walls and root surface strain. They appear more frequently when high-taper instruments are used or in teeth with curved roots, as the part of the canal with the maximum curvature accumulates the maximum stress. ${ }^{4}$ The importance of this topic consists in the fact that vertical root fractures may initiate from dentinal cracks, and eventually necessitate tooth extraction. ${ }^{5}$

2Shape (2S; Micro-Mega, Besançon, France) instruments with sequential rotary systems are manufactured by means of specific heat treatment (T-Wire), which is believed to provide higher flexibility and cyclic fatigue resistance, and preserve the elasticity of NiTi files. ${ }^{6}$

EdgeFile $^{\circledR}$ X1 (EFX1; EdgeEndo, Albuquerque, USA) is a reciprocating system manufactured from the annealed heat-treated NiTi alloy, commercially known as FireWire $^{\mathrm{TM}}$. Its manufacturer claims that it has a high torque strength, optimal flexibility and an increased cyclic fatigue resistance. ${ }^{7}$

NeoNiTi (NN; Neolix, Châtres-la-Forêt, France) is a single-file system with a full-rotational motion that benefits from the electric discharge machining (EDM) technology. Its manufacturer claims that it has optimal flexibility, a high fracture resistance, and a favorable shaping ability due to its unique properties, rectangular cross-section, efficient cutting blades, and built-in abrasive surface. ${ }^{8}$

Micro-computed tomography (micro-CT) is an imaging modality suggested for the assessment of dentin and its changes without damaging the tooth structure. Due to its high accuracy, it is commonly used for the assessment of dentinal microcrack formation following root canal instrumentation. ${ }^{2,3,9,10}$

This study aimed to assess the formation of new dentinal microcracks following the instrumentation of curved root canals of mandibular molars with the 2S, EFX1 and NN systems by using micro-CT.

\section{Material and methods}

The study protocol was approved by the Research Ethics Committee of Shahid Beheshti University of Medical Sciences, Tehran, Iran (IR.SBMU.DRC.REC.1399.036).

\section{Sample size calculation}

The sample size was calculated to be 10 in each group (to assess the effect of instruments on dentin), assuming the effect size of 7.6 for the microcracks reported after root canal instrumentation in previous studies, ${ }^{11,12}$ $\alpha=0.05, \beta=0.95$, and a study power of $90 \%$, using the PASS 15 software (NCSS, Kaysville, USA).

\section{Sample selection}

A total of 250 mandibular first and second molars were evaluated for inclusion in this study. All roots were inspected under a stereomicroscope at $\times 12$ magnification. Teeth with immature apices and external dentinal defects were excluded. Next, the teeth underwent highresolution cone-beam computed tomography (CBCT) with the use of a NewTom ${ }^{\circledR}$ VGi scanner (NewTom, Imola, Italy) with the exposure parameters of $110 \mathrm{kVp}$, $9.5 \mathrm{~mA}$, a voxel size of $0.1 \mathrm{~mm}$, and a field of view (FoV) of $6 \times 6 \mathrm{~cm}$. The $\mathrm{NNT}^{\circledR}$ viewer, v. 8.0 (NewTom) was used to assess different sections in order to classify the root canal system and root canal curvature. Root canal curvature was measured according to Schneider's method. ${ }^{13}$ In this method, the degree of root canal curvature is determined by measuring the angle formed between the longitudinal axis of the root canal and a line drawn from the initiation point of curvature to the apical foramen. Molar teeth with a Vertucci type IV mesial root, a mesiobuccal root canal curvature of $20-40^{\circ}$ on sagittal and/or coronal sections, no calcification, no internal or external root resorption, and no history of previous endodontic treatment were enrolled. The mesiobuccal canals of the mesial roots of 30 mandibular molars that met the eligibility criteria were selected. ${ }^{14}$ All teeth were stored in a $0.1 \%$ thymol solution during the course of the study.

The crowns and distal roots of all teeth were cut with a low-speed saw (IsoMet ${ }^{\text {TM }}$ 4000; Buehler Ltd., Lake Bluff, USA) under water cooling, and all remaining roots were standardized to have a length of $12 \pm 1 \mathrm{~mm}$ from the apex.

To simulate the periodontal ligament, the root surfaces were wrapped with 1 layer of aluminum foil and mounted in tubes filled with an acrylic resin (Kulzer, Hanau, Germany). After setting, the aluminum foil wrap was removed from the root surfaces and the created space was filled with a silicon impression material (GC Corporation, Tokyo, Japan). The roots were immediately placed back in the block. ${ }^{12}$

Each root was randomly assigned to one of the 3 groups of $2 \mathrm{~S}$, EFX1 or $\mathrm{NN}(n=10)$ for root canal instrumentation. All roots underwent micro-CT (LOTUS-inVivo; Behin Negareh Co., Tehran, Iran) prior to instrumentation ${ }^{15}$ and 400-500 micro-CT transverse cross-sectional images were obtained for each root. The exposure parameters included an isotropic resolution of $31 \mu \mathrm{m}$, a voltage of $99 \mathrm{kV}$, 
an amperage of $88 \mu \mathrm{A}$, and a frame exposure time of $2 \mathrm{~s}$, with an aluminum filter with a thickness of $0.5 \mathrm{~mm}$, a rotation of $360^{\circ}$ and a rotation step of $0.3^{\circ}$.

\section{Root canal preparation}

The working length was determined by introducing a size $10 \mathrm{~K}$-file (Mani Inc., Utsunomiya, Japan) into the root canal until its tip was visible at the apical foramen; $1 \mathrm{~mm}$ was subtracted from this length to determine the working length. Next, a glide path was created using a size 15 K-file (Mani Inc.). Instrumentation was performed by the same operator in all groups.

Each file was used for the instrumentation of 2 canals, using an endomotor (Silver; VDW, Munich, Germany) with the speed and torque set as recommended by the manufacturer.

In the $2 \mathrm{~S}$ group, TS1 $(25,04)$ and TS2 $(25,06)$ files were sequentially introduced into the canal with a torque of $1.5 \mathrm{~N} \cdot \mathrm{cm}$ and a speed of $300 \mathrm{rpm}$ with a pecking motion, and proceeded with an up-and-down motion until the file reached the working length.

In the EFX1 group, the EdgeFile X1 instrument $(25,06)$ was used with a reciprocating motion at the WaveOne setting until it reached the working length.

In the NN group, the NeoNiTi A1 instrument $(25,06)$ with a torque of $1.5 \mathrm{~N} \cdot \mathrm{cm}$ and a speed of $300 \mathrm{rpm}$ was used with a circumferential brushing motion until reaching the working length.

In all systems, after 3 pecking motions, the file was removed from the canal and its flutes were cleaned with gauze. Next, the root canal was rinsed with $2 \mathrm{~mL}$ of $2.5 \%$ sodium hypochlorite. ${ }^{2}$ After root canal instrumentation, the root canals underwent micro- $\mathrm{CT}$ again with the same exposure parameters as those used for pre-instrumentation scanning.

\section{Assessment of dentinal microcracks}

The reconstructed micro-CT images were transferred to a data viewer (RadiAnt DICOM Viewer 2020.2). The pre- and post-instrumentation micro-CT cross-sectional images $(N=29,280)$ were simultaneously evaluated.
Two blinded observers (endodontists) with 4 years of clinical experience evaluated all sections twice at a 2-week interval (to assess intra-observer agreement) and recorded the microcracks. A microcrack was defined as any incomplete crack line initiating from the canal wall and extending into the dentin structure, but not reaching the external surface of the root. A complete crack was defined as a crack line initiating from the root canal wall and extending to the external surface of the root. A craze line was defined as any other line that did not reach the root surface or extended from the external surface of the root into the dentin, but did not reach the root canal wall. ${ }^{11}$ In case of any disagreement between the observers, the images were inspected again until consensus was reached.

\section{Statistical analysis}

Cohen's kappa coefficient ( $\mathrm{k}$ ) was calculated to assess inter- and intra-observer reliability. The number of dentinal cracks before and after instrumentation in each group was compared by means of McNemar's test using the IBM SPSS Statistics for Windows software , v. 25.0 (IBM Corp., Armonk, USA), at a level of significance of 0.05 .

\section{Results}

The kappa coefficient was calculated to be 0.86 , which indicated very good inter-observer agreement (reliability). The kappa coefficient for intra-observer agreement (reliability) was found to be 1 for both observers, indicating complete agreement.

Out of the 29,280 cross-sectional images evaluated in this study, $11.5 \%$ showed dentinal microcracks $(n=3,362)$. On the post-instrumentation images, the frequency percentage of microcracks was $12.0 \%(n=585)$ in the $2 \mathrm{~S}$ group, $8.8 \%(n=402)$ in the EFX1 group and $13.3 \%$ $(n=694)$ in the NN group. All the microcracks detected on the post-instrumentation images were also present on the pre-instrumentation images and no new microcracks were formed after root canal instrumentation with the aforementioned systems $(p=1.00)$ (Table 1$)$.

Table 1. Dentinal microcracks on the cross-sectional images of the roots before and after instrumentation

\begin{tabular}{|c|c|c|c|c|c|c|c|c|c|}
\hline \multirow{3}{*}{$\begin{array}{l}\text { Scanning } \\
\text { time }\end{array}$} & \multicolumn{9}{|c|}{ Dentinal microcracks } \\
\hline & \multicolumn{3}{|c|}{$2 S$} & \multicolumn{3}{|c|}{ EFX1 } & \multicolumn{3}{|c|}{ NN } \\
\hline & $\begin{array}{l}\text { presence of } \\
\text { microcracks }\end{array}$ & $\begin{array}{l}\text { absence of } \\
\text { microcracks }\end{array}$ & total & $\begin{array}{l}\text { presence of } \\
\text { microcracks }\end{array}$ & $\begin{array}{l}\text { absence of } \\
\text { microcracks }\end{array}$ & total & $\begin{array}{l}\text { presence of } \\
\text { microcracks }\end{array}$ & $\begin{array}{l}\text { absence of } \\
\text { microcracks }\end{array}$ & total \\
\hline $\begin{array}{l}\text { Before } \\
\text { instrumentation }\end{array}$ & $\begin{array}{c}585 \\
(12.0)\end{array}$ & $\begin{array}{l}4,295 \\
(88.0)\end{array}$ & $\begin{array}{l}4,880 \\
(100)\end{array}$ & $\begin{array}{l}402 \\
(8.8)\end{array}$ & $\begin{array}{l}4,149 \\
(91.2)\end{array}$ & $\begin{array}{l}4,551 \\
(100)\end{array}$ & $\begin{array}{c}694 \\
(13.3)\end{array}$ & $\begin{array}{l}4,515 \\
(86.7)\end{array}$ & $\begin{array}{l}5,209 \\
(100)\end{array}$ \\
\hline $\begin{array}{l}\text { After } \\
\text { instrumentation }\end{array}$ & $\begin{array}{c}585 \\
(12.0)\end{array}$ & $\begin{array}{l}4,295 \\
(88.0)\end{array}$ & $\begin{array}{l}4,880 \\
(100)\end{array}$ & $\begin{array}{l}402 \\
(8.8)\end{array}$ & $\begin{array}{l}4,149 \\
(91.2)\end{array}$ & $\begin{array}{l}4,551 \\
(100)\end{array}$ & $\begin{array}{c}694 \\
(13.3)\end{array}$ & $\begin{array}{l}4,515 \\
(86.7)\end{array}$ & $\begin{array}{l}5,209 \\
(100)\end{array}$ \\
\hline Total & $\begin{array}{l}1,170 \\
(12.0)\end{array}$ & $\begin{array}{l}8,590 \\
(88.0)\end{array}$ & $\begin{array}{l}9,760 \\
(100)\end{array}$ & $\begin{array}{l}804 \\
(8.8)\end{array}$ & $\begin{array}{l}8,298 \\
(91.2)\end{array}$ & $\begin{array}{l}9,102 \\
(100)\end{array}$ & $\begin{array}{l}1,388 \\
(13.3)\end{array}$ & $\begin{array}{l}9,030 \\
(86.7)\end{array}$ & $\begin{array}{c}10,418 \\
(100)\end{array}$ \\
\hline
\end{tabular}

Data presented as number (percentage) ( $(\%))$. 2S - 2Shape; EFX1 - EdgeFile X1; NN - NeoNiTi. 
Figures 1-4 show the cross-sectional images of the roots before and after instrumentation. Table 2 presents the total frequency percentage of microcracks on the cross-sectional images of the roots in all groups. Figure 5 shows the sequence of cross-sectional images of the same mesial root, demonstrating the development of microcracks.

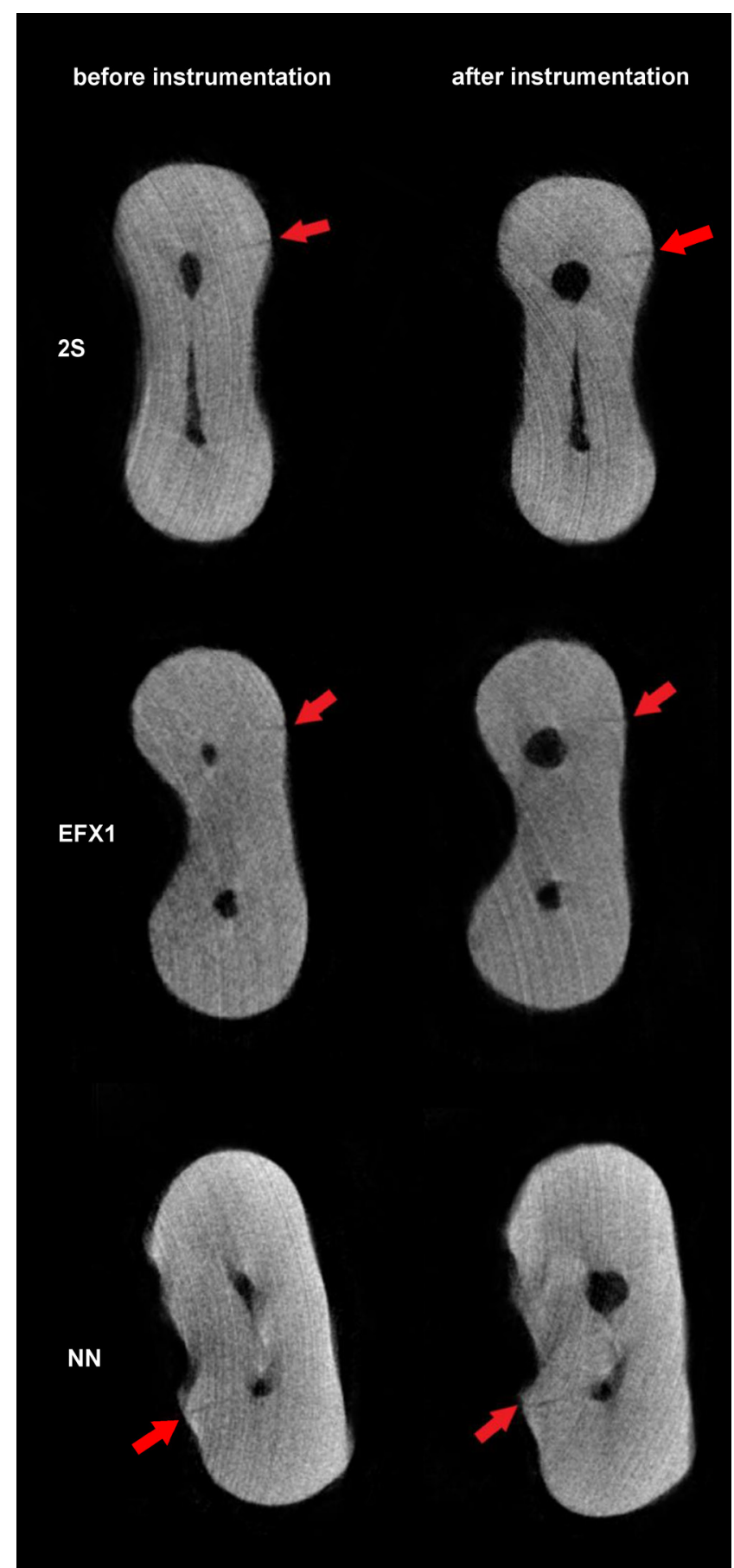

Fig. 1. Representative cross-sectional images of the mesial roots of mandibular molars showing the presence of cracks (arrows) before and after the preparation of the mesiobuccal canals with the 2Shape (2S), EdgeFile X1 (EFX1) and NeoNiTi (NN) systems

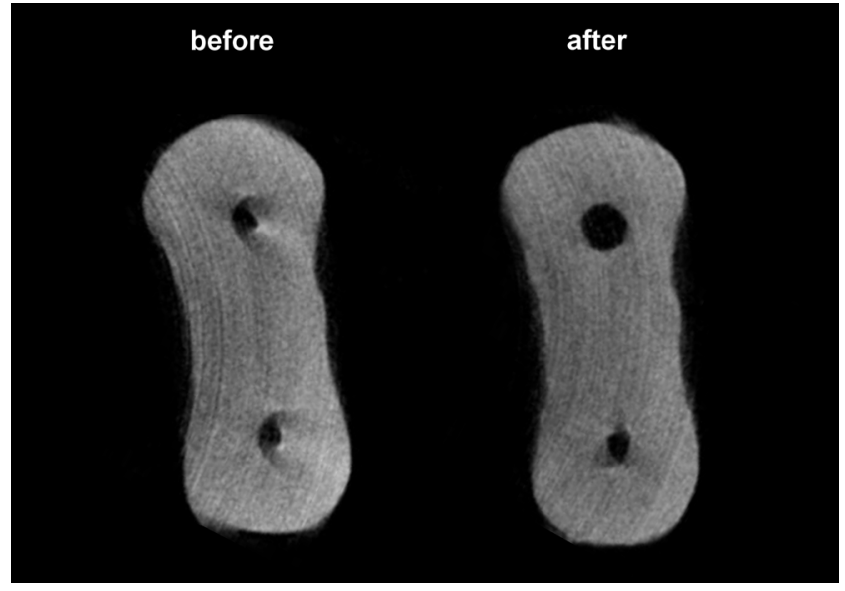

Fig. 2. Representative cross-sectional images of the roots, showing the absence of cracks before and after instrumentation

Table 2. Total frequency of microcracks on the cross-sectional images of the roots in all 3 study groups

\begin{tabular}{|l|c|c|c|} 
Scanning time & $\begin{array}{c}\text { Presence } \\
\text { of microcracks }\end{array}$ & $\begin{array}{c}\text { Absence } \\
\text { of microcracks }\end{array}$ & Total \\
\hline $\begin{array}{l}\text { Before } \\
\text { instrumentation }\end{array}$ & 1,681 & 12,959 & 14,640 \\
After & $(11.5)$ & $(88.5)$ & $(100)$ \\
instrumentation & 1,681 & 12,959 & 14,640 \\
Total & $(11.5)$ & $(88.5)$ & $(100)$ \\
& 3,362 & 25,918 & 29,280 \\
& $(11.5)$ & $(88.5)$ & $(100)$ \\
\hline
\end{tabular}

Data presented as $n(\%)$.

\section{Discussion}

Mandibular molars have shown a mean root canal curvature $>20^{\circ}$ in morphological studies. ${ }^{16,17}$ However, many studies on dentinal microcracks in mandibular molars have evaluated teeth with a mesial root curvature of $10-20^{\circ} .9,10,18$ Thus, mandibular molars with a mesial root curvature $>20^{\circ}$ were used in this study.

This study evaluated the effects of the sequential rotary (2S), reciprocating (EFX1) and single-file rotational (NN) systems on microcrack formation in the curved root canals of mandibular molars. Although dentinal microcracks were observed in all 3 groups after root canal instrumentation, these microcracks were present on the micro-CT images obtained before root canal instrumentation as well. Thus, no new microcracks were formed after root canal instrumentation with the 2S, EFX1 and NN systems. This finding is in agreement with the results of the majority of previous studies that used micro-CT for the assessment of microcrack formation after root canal instrumentation. ${ }^{9,10,12,19-25}$ A study conducted in 2014 used micro-CT for the first time and showed no cause-and-effect relationship between dentinal microcrack formation and root canal instrumentation. ${ }^{10}$ After that, many micro-CT studies confirmed this finding. In their study on human cadavers, De-Deus et al. evaluated 19,060 cross-sectional micro-CT images of maxillary first 
before instrumentation
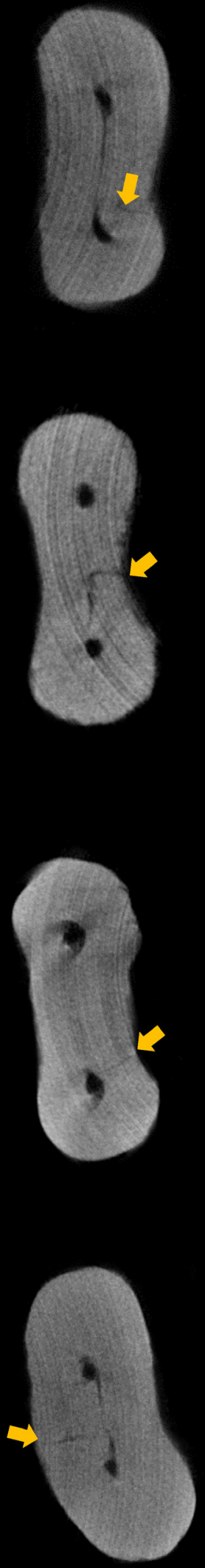

after instrumentation
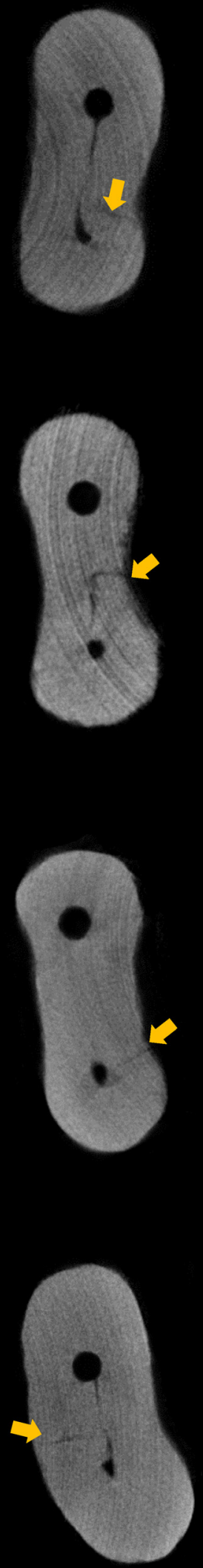

before instrumentation

after instrumentation
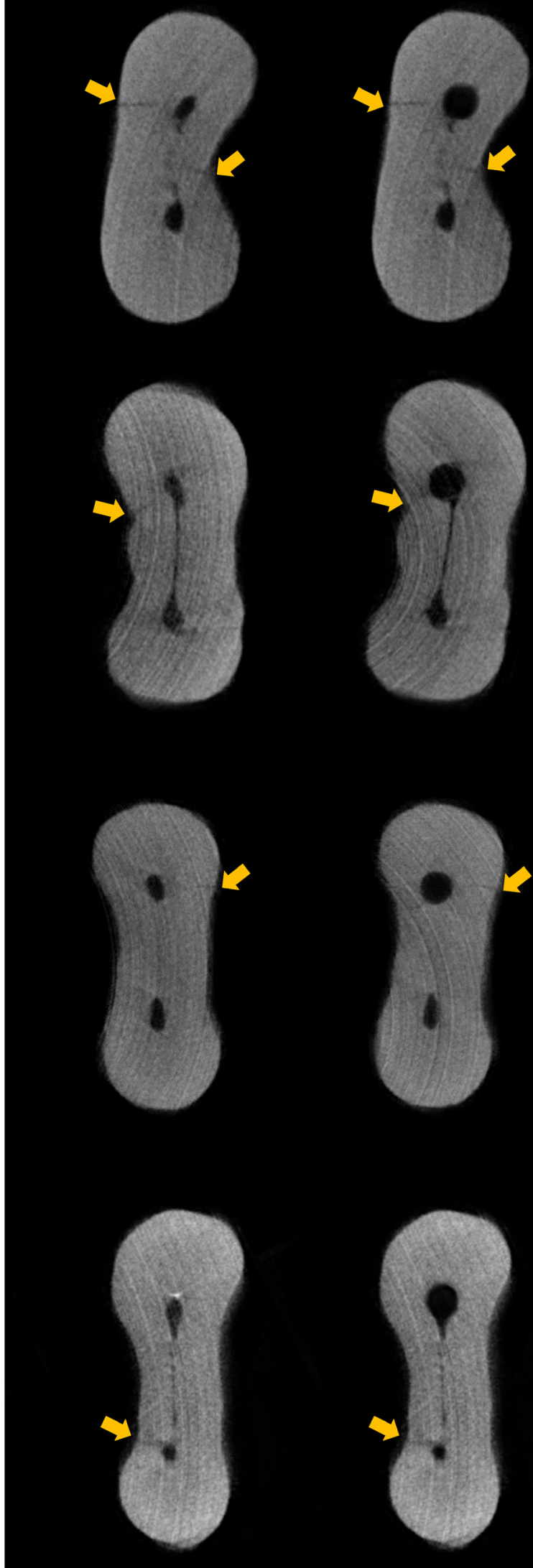

Fig. 3. Representative cross-sectional images of the mesial roots of mandibular molars, showing the presence of cracks (arrows) before and after the preparation of the mesiobuccal canals. 


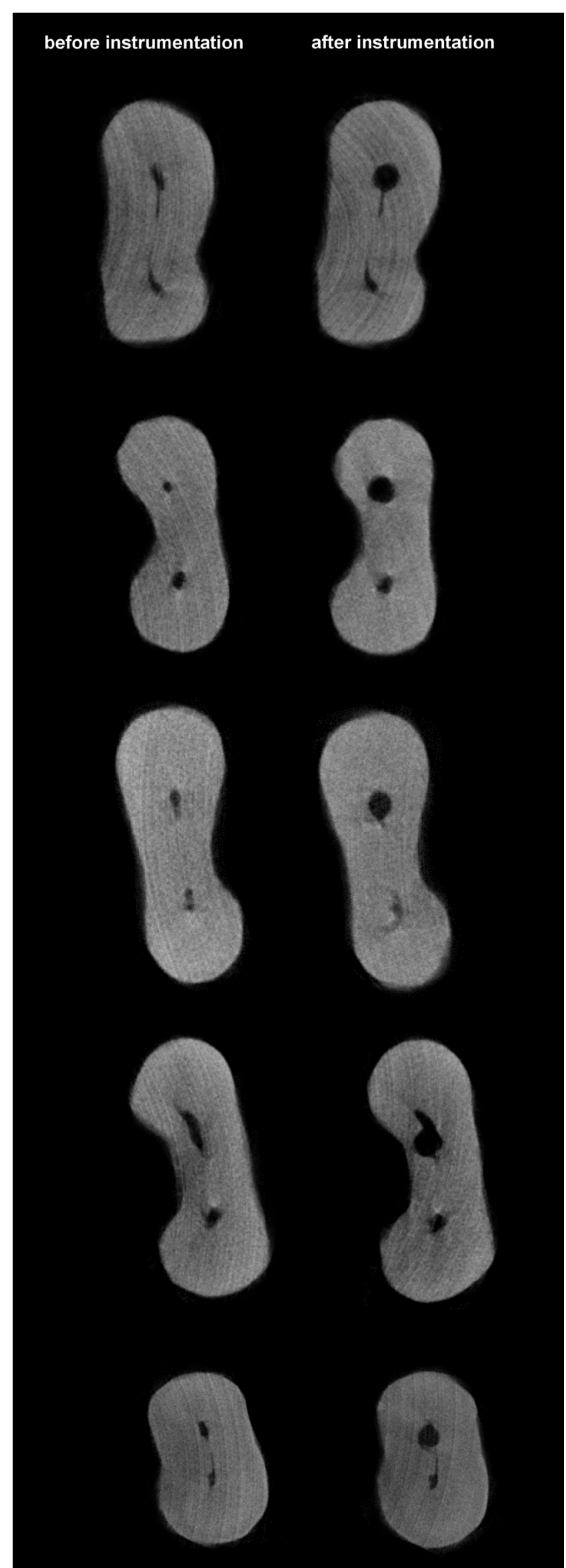

Fig. 4. Representative cross-sectional images of the roots, showing the absence of cracks before and after instrumentation and second premolars and observed that root canal preparation with rotary and Reciproc files did not cause new dentinal microcracks. ${ }^{21}$ In another study, De-Deus et al. evaluated the effects of the ProTaper Next and Twisted File Adaptive systems on dentinal microcrack formation with the use of micro-CT. ${ }^{9}$ They evaluated 25,820 crosssectional images of the mesial roots of mandibular molars before and after instrumentation and found no new microcracks after instrumentation. ${ }^{9}$ Another recent study compared root canal preparation with the ProTaper Next, ProTaper Gold and WaveOne Gold systems with regard to the formation of dentinal microcracks in mandibular molars with a root curvature $>20^{\circ} .{ }^{12}$ All the microcracks detected after instrumentation on micro-CT images were present on the pre-instrumentation images as well, and no new microcracks were observed. ${ }^{12}$ An in vivo study on intact premolars scheduled for extraction as part of orthodontic treatment evaluated with micro-CT the effects of manual instrumentation and rotary instrumentation with ProTaper files. ${ }^{22}$ They found no new dentinal microcracks after root canal instrumentation. ${ }^{22}$

On the contrary, some micro-CT studies have reported the formation of new microcracks after root canal preparation. ${ }^{2,3,18,26}$ Ceyhanli et al. showed an increase in the number of dentinal microcracks after the preparation of the mesial roots of mandibular molars with all 3 systems of ProTaper Universal, RaCe and SafeSider, using micro$\mathrm{CT} .^{2}$ They reported the maximum number of microcracks in the ProTaper Universal group. ${ }^{2}$ Also, Li et al. demonstrated an increase in the length of microcracks present in the mesial roots of mandibular first molars with a curvature of $10-20^{\circ}$ after root canal instrumentation. ${ }^{18}$ They used single-file systems and showed that the change in microcrack length in the OneShape group was greater than that in the WaveOne and Reciproc systems. ${ }^{18}$ Bayram et al. determined that root canal instrumentation with ProTaper Universal significantly increased the formation of new dentinal microcracks. ${ }^{3}$ Jamleh et al. reported that both the WaveOne reciprocating and ProTaper Universal rotary systems resulted in the formation of new microcracks. ${ }^{26}$

Controversy in the results may be related to differences in the methodologies and quality of tools used. For instance, in the study by Ceyhanli et al., ${ }^{2}$ the reason for the increase in the number of microcracks might be the fact that only 10 root sections were evaluated $(<1.5 \%$ of all the obtained images); this may have led to false positive results due to the presence of noise or artifacts. ${ }^{27}$ Jamleh et al. required dry and dehydrated surfaces to measure root surface strain as well as for the application of the staining technique, ${ }^{26}$ which increased the risk of formation of defects and dentinal microcracks. ${ }^{28}$ Another influential factor in microcrack formation is the working length of the root canal. A recently published systematic review indicated that in all root canal preparation techniques, a proper working length ( $1 \mathrm{~mm}$ short of the apex) is required to prevent microcrack formation. ${ }^{23}$ This was 


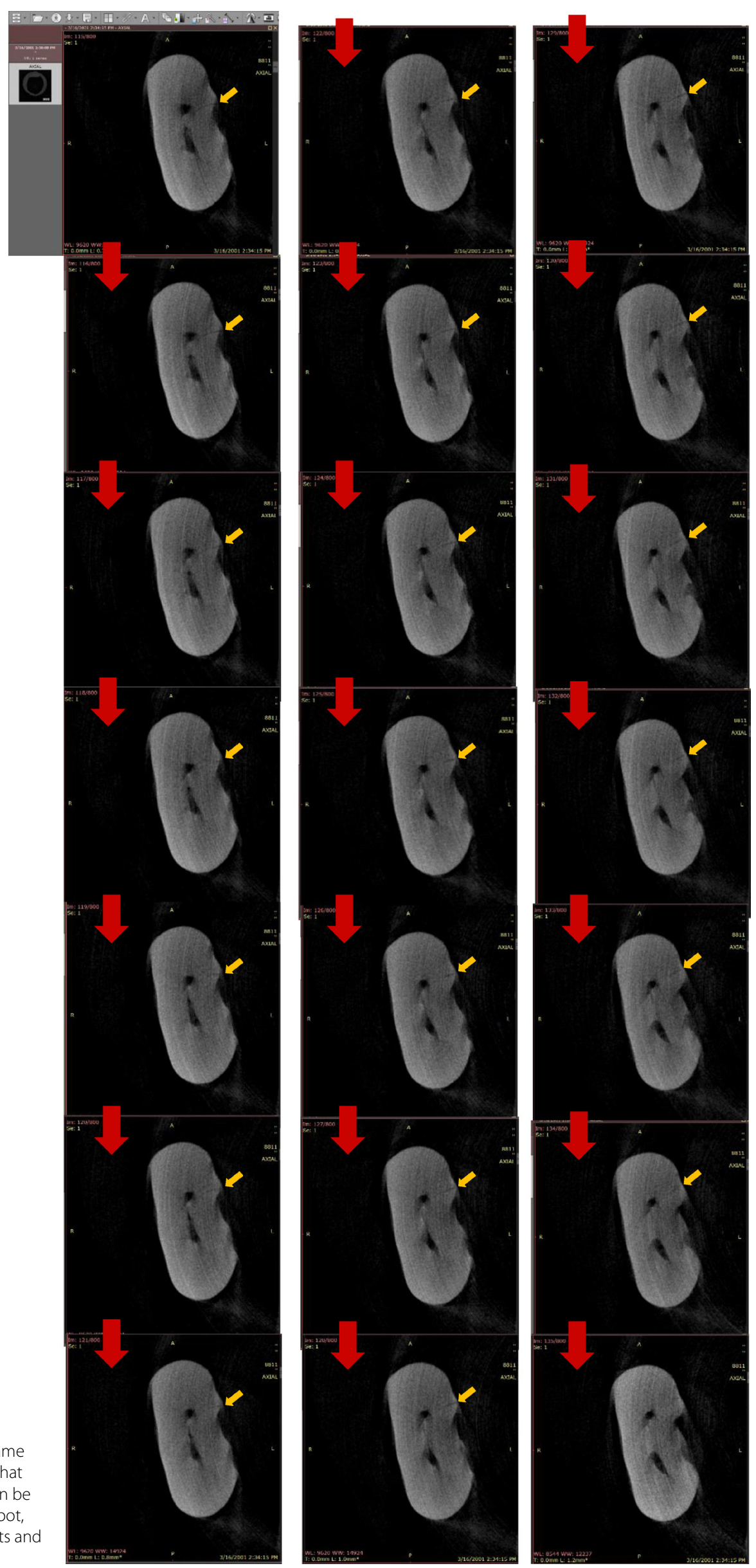

Fig. 5. Sequence of cross-sectional images of the same mesial root of a mandibular molar, demonstrating that the development of microcracks (yellow arrows) can be evaluated throughout the longitudinal axis of the root confirming the identification of true dentinal defects and artifacts 
not the case in the studies by Li et al. ${ }^{18}$ and Bayram et al., ${ }^{3}$ and might be the possible reason for the development of dentinal microcracks in their studies.

Studies that used the sectioning technique for the assessment of dentinal microcracks have reported controversial results. The majority of such studies have reported the formation of dentinal microcracks following the use of different instrumentation techniques., ${ }^{4,23}$ However, there is no consensus among such studies, and the prevalence of microcrack formation following the use of different instrumentation techniques ranges from $0 \%$ to $80 \%{ }^{4}$ According to Versiani et al., such a wide range of variation in the prevalence of microcracks may be attributed to the use of different systems and root canal preparation protocols, methods of observation of dentinal defects (with or without a dye), root canal anatomy, the irrigation protocol, and the type of classification used for defects. ${ }^{4}$ In sectioning studies, $3-4$ sections of the root are often evaluated, made at $1,3,7$, and $9 \mathrm{~mm}$, or 2, 4, 6, and $8 \mathrm{~mm}$ from the apex. This is among the most important drawbacks of the sectioning technique, as the assessment of such a limited number of slices increases the risk of missing a high number of defects along the root canal wall. However, with micro-CT, hundreds of slices of each root can be evaluated. Moreover, micro-CT has a much higher resolution than stereomicroscopy. ${ }^{5}$ Another important drawback is the destructive nature of the sectioning method. Stringheta et al. compared the sectioning and micro-CT methods for the detection of dentinal microcracks after root canal instrumentation..$^{29}$ They inspected the micro-CT scans before sectioning and noticed that the sectioning method was destructive, and resulted in the formation of new dentinal defects. ${ }^{29}$ For this reason, the majority of studies that used the sectioning technique have reported new dentinal crack formation after the instrumentation process. ${ }^{30}$ The advantages of micro-CT include its non-destructive nature, its ability to exactly locate dentinal cracks in the root and its three-dimensional (3D) assessment. This allows the evaluation of hundreds of cross-sectional images of the entire root length and the detection of microcracks already present in the root canal walls prior to instrumentation or at different stages of endodontic treatment. ${ }^{9,24}$

The kinematics of the instrument motion and its effect on the formation of dentinal microcracks is another topic of interest that has been addressed in previous studies. A meta-analysis that mainly focused on sectioning studies reported that a reciprocating motion produced a significantly smaller number of dentinal cracks as compared to a rotational motion. ${ }^{30}$ However, 2 recently published systematic reviews on micro-CT studies found no correlation between instrumentation kinematics and the formation of dentinal microcracks, ${ }^{23,24}$ which confirms the findings of the present study regarding no correlation between the type of instrument motion and dentinal microcrack formation.

\section{Conclusions}

According to the results of this in vitro study, the instrumentation of curved root canals with the 2S, EFX1 and $\mathrm{NN}$ systems does not result in the formation of new dentinal microcracks.

\section{ORCID iDs}

Yazdan Shantiaee (D) https://orcid.org/0000-0002-9189-2184 Babak Zandi (iD https://orcid.org/0000-0001-5465-2117

Shiva Shojaeian (1) https://orcid.org/0000-0002-4915-6028

Niloofar Mortezapour (D) https://orcid.org/0000-0002-9202-774X

Fatemeh Soltaninejad (1) https://orcid.org/0000-0001-9698-2077

\section{References}

1. Schilder H. Cleaning and shaping the root canal. Dent Clin North Am. 1974;18(2):269-296. PMID:4522570.

2. Ceyhanli KT, Erdilek N, Tatar I, Celik D. Comparison of ProTaper, RaCe and Safesider instruments in the induction of dentinal microcracks: A micro-CT study. Int Endod J. 2016;49(7):684-689. doi:10.1111/iej.12497

3. Bayram HM, Bayram E, Ocak M, Uygun AD, Celik HH. Effect of ProTaper Gold, Self-Adjusting File, and XP-endo Shaper instruments on dentinal microcrack formation: A micro-computed tomographic study. J Endod. 2017;43(7):1166-1169. doi:10.1016/j.joen.2017.02.005

4. Versiani MA, Souza E, De-Deus G. Critical appraisal of studies on dentinal radicular microcracks in endodontics: Methodological issues, contemporary concepts, and future perspectives. Endod Topics. 2015;33(1):87-156. doi:10.1111/etp.12091

5. Wilcox LR, Roskelley C, Sutton T. The relationship of root canal enlargement to finger-spreader induced vertical root fracture. J Endod. 1997;23(8):533-534. doi:10.1016/S0099-2399(97)80316-0

6. Micro-Mega. The 2Shape brochure. https://micro-mega.com/wpcontent/uploads/2018/03/60301807-C_Brochure-2Shape_EN_WEB. pdf. Accessed November 19, 2020.

7. EdgeEndo. The EdgeFile ${ }^{\circledR} \mathrm{X} 1$ directions for use. https://edgeendo. com/wp-content/uploads/2015/08/DFU-EdgeFile-x1.pdf. Accessed November 19, 2020.

8. Neolix. The NeoNiTi instruction manual. https://www.neolix.eu/ themes/neo/assets/img/pdf/flyer/FLYER-NEONITI-ENGLISH.pdf. Accessed November 19, 2020.

9. De-Deus G, Belladonna FG, Souza EM, et al. Micro-computed tomographic assessment on the effect of ProTaper Next and Twisted File Adaptive systems on dentinal cracks. J Endod. 2015;41(7):1116-1119. doi:10.1016/j.joen.2015.02.012

10. De-Deus G, Nogueira Leal Silva EJ, Marins J, et al. Lack of causal relationship between dentinal microcracks and root canal preparation with reciprocation systems. J Endod. 2014;40(9):1447-1450. doi:10.1016/j.joen.2014.02.019

11. Bürklein S, Tsotsis $P$, Schäfer E. Incidence of dentinal defects after root canal preparation: Reciprocating versus rotary instrumentation. J Endod. 2013;39(4):501-504. doi:10.1016/j.joen.2012.11.045

12. Miguéns-Vila R, Martín-Biedma B, De-Deus G, Belladonna F, PeñaLópez A, Castelo-Baz P. Micro-computed tomography evaluation of dentinal micro-cracks after preparation of curved root canals with ProTaper Gold, WaveOne Gold and ProTaper Next instruments. J Endod. 2021;47(2):309-314. doi.org/10.1016/j.joen.2020.10.014

13. Schneider SW. A comparison of canal preparations in straight and curved root canals. Oral Surg Oral Med Oral Pathol. 1971;32(2):271-275. doi:10.1016/0030-4220(71)90230-1

14. Kırıcı D, Demirbuga S, Karataş E. Micro-computed tomographic assessment of the residual filling volume, apical transportation, and crack formation after retreatment with Reciproc and Reciproc Blue systems in curved root canals. J Endod. 2020;46(2):238-243. doi:10.1016/j.joen.2019.11.003

15. Fouladi M, Gholami K, Ghadiri H. LOTUS-inVivo micro computed tomography system for imaging of small animals and ex-vivo biological samples. Frontiers Biomed Technol. 2020;7(2):134-137. doi:10.18502/fbt.v7i2.3860 
16. Gu Y, Lu Q, Wang P, Ni L. Root canal morphology of permanent threerooted mandibular first molars: Part II - measurement of root canal curvatures. J Endod. 2010;36(8):1341-1346. doi:10.1016/j.joen.2010.04.025

17. Schäfer E, Diez C, Hoppe W, Tepel J. Roentgenographic investigation of frequency and degree of canal curvatures in human permanent teeth. J Endod. 2002;28(3):211-216. doi:10.1097/00004770200203000-00017

18. Li ML, Liao WL, Cai HX. A micro-computed tomographic evaluation of dentinal microcrack alterations during root canal preparation using single-file Ni-Ti systems. Exp Ther Med. 2018;15(1):494-499. doi:10.3892/etm.2017.5399

19. Bayram HM, Bayram E, Ocak M, Uzuner MB, Geneci F, Celik HH. Micro-computed tomographic evaluation of dentinal microcrack formation after using new heat-treated nickel-titanium systems. J Endod. 2017;43(10):1736-1739. doi:10.1016/j.joen.2017.05.024

20. de Oliveira BP, Câmara AC, Duarte DA, Heck RJ, Dantas Antonino AC, Aguiar CM. Micro-computed tomographic analysis of apical microcracks before and after root canal preparation by hand, rotary, and reciprocating instruments at different working lengths. J Endod. 2017:43(7):1143-1147. doi:10.1016/j.joen.2017.01.017

21. De-Deus G, de Azevedo Carvalhal JC, Belladonna FG, et al. Dentinal microcrack development after canal preparation: A longitudinal in situ micro-computed tomography study using a cadaver model. J Endod. 2017;43(9):1553-1558. doi:10.1016/j.joen.2017.04.027

22. PradeepKumar AR, Shemesh $H$, Archana D, et al. Root canal preparation does not induce dentinal microcracks in vivo. J Endod. 2019;45(10):1258-1264. doi:10.1016/j.joen.2019.06.010

23. Valle AD, Dotto L, Morgental RD, Pereira-Cenci T, da Rocha Pereira GK, Sarkis-Onofre R. Influence of root canal preparation on formation of dentinal microcracks: A systematic review. Braz Dent J. 2020;31(3):201-220. doi:10.1590/0103-6440202002652

24. Nagendrababu V, Aly Ahmed HM. Shaping properties and outcomes of nickel-titanium rotary and reciprocation systems using micro-computed tomography: A systematic review. Quintessence Int. 2019;50(3):186-195. doi:10.3290/j.qi.a41977

25. Oliveira Vieira ML, Dantas HV, de Sousa FB, et al. Morphologic changes of apical foramen and microcrack formation after foraminal enlargement: A scanning electron microscopic and microcomputed tomographic analysis. J Endod. 2020;46(11):1726-1732. doi:10.1016/j.joen.2020.07.017

26. Jamleh A, Komabayashi T, Ebihara A, et al. Root surface strain during canal shaping and its influence on apical microcrack development: A preliminary investigation. Int Endod J. 2015;48(12):1103-1111. doi:10.1111/iej.12406

27. De-Deus G, Belladonna FG, Silva E, Souza EJNL, Versiani MA. Critical appraisal of some methodological aspects of using micro-CT technology in the study of dentinal microcracks in endodontics. Int Endod J. 2016;49(2):216-219. doi:10.1111/iej.12570

28. Shemesh $H$, Lindtner T, Portoles CA, Zaslansky P. Dehydration induces cracking in root dentin irrespective of instrumentation: A two-dimensional and three-dimensional study. J Endod. 2018;44(1):120-125. doi:10.1016/j.joen.2017.07.025

29. Stringheta $C P$, Pelegrine RA, Kato AS, et al. Micro-computed tomography versus the cross-sectioning method to evaluate dentin defects induced by different mechanized instrumentation techniques. J Endod. 2017;43(12):2102-2107. doi:10.1016/j.joen.2017.07.015

30. Wei $X$, Hu B, Peng $H$, Tang M, Song J. The incidence of dentinal cracks during root canal preparations with reciprocating singlefile and rotary-file systems: A meta-analysis. Dent Mater J. 2017;36(3):243-252. doi:10.4012/dmj.2016-208 